\title{
Editorial
}

\section{Zukunft mit Komplementärmedizin - Die Umsetzung des neuen Verfassungsartikels 118a)}

Mit einer wuchtigen Zweidrittelsmehrheit hat das Schweizer Volk am 17. Mai der Verankerung der Komplementärmedizin in der Eidgenössischen Verfassung zugestimmt - ein Ereignis, das weit über die Landesgrenzen hinaus Beachtung findet. Doch wie soll der neue Verfassungsartikel in die Praxis umgesetzt werden?

Es geht nicht primär um aufwändige Gesetzesrevisionen, die bestehenden Gesetze genügen weitgehend. Es braucht jetzt einzig politischen Willen, die ungebrochene Aufmerksamkeit der Bürger und die Kooperationsbereitschaft der zuständigen Ämter und der medizinischen Fakultäten. Und es ist auch kein Kostenschub zu befürchten; selbst die Krankenkassen haben mittlerweile bestätigt, dass Komplementärmedizin günstig ist.

Die Wiederaufnahme der fünf ärztlichen Fachrichtungen Anthroposophische Medizin, Homöopathie, Neuraltherapie, Phytotherapie, Traditionelle Chinesische Medizin in die obligatorische Krankenversicherung nach dem Rauswurf durch Bundesrat Couchepin 2005 ist an sich bereits angebahnt. Alle Fachschaften haben beim Bundesamt für Gesundheit (BAG) Anmeldungen für neue Anträge deponiert. In den letzten vier Jahren sind zusätz-

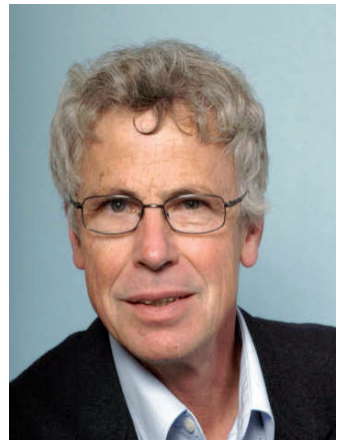
liche gute klinische Studien publiziert worden. Es besteht aber weiterhin die Schwierigkeit, dass sich weder Bundesrat noch BAG auf verbindliche Beurteilungskriterien festlegen wollen. In seiner Antwort an die Geschäftsprüfungskommission des Nationalrats vom 24.6.2009 stellt der Bundesrat diesbezüglich nur fest: „Der Bundesrat teilt die Ansicht, dass die WZW-Kriterien zu konkretisieren und operationalisieren sind.“ Das neue „Handbuch zur Antragstellung auf Kostenübernahme bei neuen oder umstrittenen Leistungen" des BAG vom Januar 2008 bleibt in seinen Vorgaben ebenfalls vage. Das Antragsverfahren ist grundsätzlich für konkrete Einzelleistungen vorgesehen, lässt aber offen, ob mit dem Meldeformular auch „spezielle Leistungen“ wie z.B. „Behandlungsmethoden, die sehr breit und unabhängig von spezifischen Indikationen eingesetzt werden, oder ein ganzes Therapiesystem" erfasst werden können. Kurz: Weitere Willkürentscheide sind vorprogrammiert!

Zur Wahrung des bewährten Heilmittelschatzes hat das Heilmittelgesetz (HMG) von 2002 an sich längst die Möglichkeit für vereinfachte Zulassungen geschaffen, nur hat die Swissmedic solche Erleichterungen bisher nur ungenügend umgesetzt. Die Revision des HMG muss hier die Verbindlichkeit verstärken. Gleichzeitig braucht die Swissmedic aber zwingend wieder ein taugliches Gremium für die Beurteilung der Heilmittel der Komplementärmedizin. Zudem muss die komplementärmedizinische Pharmazie an den Universitäten verankert werden.

Die Integration der Komplementärmedizin in Lehre und Forschung verlangt die Schaffung von vollwertigen Lehrstühlen an den Universitäten. Hierzu sind vor allem die Kantone gefordert. Wenn zwei Drittel der Bevölkerung Komplementärmedizin beanspruchen, müssen auch die Schulmediziner ein Basiswissen davon haben. Und wenn man der Komplementärmedizin ein Forschungsdefizit vorwirft, müssen akademische Strukturen zur Forschungsförderung geschaffen werden. Dieses Anliegen stösst indessen auf geradezu verbissenen Widerstand an den medizinischen Fakultäten. Bereits hat die Schweizerische Akademie der Medizinischen Wissenschaften (SAMW) in einem „Hirtenbrief“ (Schweizerische Ärztezeitung 2009;23:892-8) die Gefahr beschworen, "dass Ärztinnen und Ärzte die traditionelle Nähe zu den wissenschaftlichen Grundlagen verlieren, d.h. die Ergebnisse der Forschung nicht im Sinne der "Evidence-based Medicine' für ihre praktisch-klinische Arbeit einsetzen..."

Für die nichtärztlichen Therapeutinnen und Therapeuten müssen jetzt zügig nationale Diplome und Vorgaben für kantonale Praxisbewilligungen geschaffen werden. Die betroffenen Berufsverbände haben sich ja in vorbildlicher Weise längst mit dem Bundesamt für Berufsbildung und Technologie (BBT) zu gemeinsamen Vorlagen zusammengefunden. Auch dieser Prozess ist durch eine Intervention des Gesundheitsministers blockiert worden.

Einen Monat nach der Volksabstimmung zur Komplementärmedizin gab Bundesrat Couchepin seinen Rücktritt bekannt. Während allgemein sein staatsmännisches Format gewürdigt wurde, hielten Volk und Parlament verbreitet Couchepin verantwortlich für das Scheitern der Gesundheitsreform in der Schweiz. Insbesondere in der sturen Abwehr gegen die Hausärzte und die Komplementärmedizin hat sich Couchepin richtiggehend verheddert. Es bleibt zu hoffen, dass die neuen Kräfte an der Spitze von EDI und BAG die Bereitschaft mitbringen werden, unter Respektierung des Volkswillens für eine qualitativ hochstehende integrative Medizin in der Schweiz einzutreten. 